\title{
ON CLOSED IDEALS OF ANALYTIC FUNCTIONS
}

\section{T. P. SRINIVASAN AND JU-KWEI WANG ${ }^{1}$}

1. The closed ideals in the algebra $A$ of all continuous functions $f\left(e^{i \theta}\right)$ on the unit circle $X=\left\{e^{i \theta}: 0 \leqq \theta<2 \pi\right\}$ which have analytic extensions $f(z),|z|<1$ have been determined by Beurling and independently by Rudin [5] as follows: Let $H^{\infty}$ denote the weak* closure $[A]_{*}$ of $A$ as a subset of $L^{\infty}(d m)$, where $m$ denotes the normalized Lebesgue measure $d \theta / 2 \pi$ on the circle. A function $q \in H^{\infty}$ is called inner if $|q|=1$ a.e. We shall regard two inner functions as the same if they are constant multiples of each other. It is well known [3, p. 66] that any such $q$ can be extended to the open unit disc to be analytic there and the extended function is of the form

$$
q(z)=z^{k} \prod_{n=1}^{\infty} \frac{z-a_{n}}{1-\bar{a}_{n} z} \frac{\bar{a}_{n}}{\left|a_{n}\right|} \cdot \exp \left[-\int_{X} \frac{e^{i \theta}+z}{e^{i \theta}-z} d \lambda(\theta)\right],
$$

where $0<\left|a_{n}\right|<1, \sum\left(1-\left|a_{n}\right|\right)<\infty$, and $\lambda$ is a non-negative measure singular relative to $m$. Then

TheOREM. For any closed ideal $N$ of $A$ there exists an inner function $q$ and a closed subset $K \subset X$ of Lebesgue measure 0 containing the points of accumulation of the zeros $a_{n}$ of $q(z)$ and the support of the singular measure $\lambda$ in the representation (1) such that $N=q Z(K)$, where

$$
Z(K)=\{f \in A: f \mid K=0\} .
$$

We offer here a proof of this theorem which was inspired by Rudin's original proof, but is relatively free of the function theoretic considerations on the circle and is therefore applicable in a more general context. In our approach we take the point of view that the closed ideals of $A$ are just the (uniformly) closed subspaces of $A$ which are invariant under multiplication by functions in $A$. The invariant subspaces of $H^{p}(d m)(1 \leqq p \leqq \infty)$ have already been characterized by us [7], also [6], following Beurling, to be of the form $q H^{p}$ for some inner function $q$; here the function $q$ is defined only a.e. but so are the functions in $H^{p}$. The functions from $A$, on the other hand, are defined everywhere and are continuous; we have therefore to take into account, in the determination of the invariant subspaces of $A$, the discontinui-

Received by the editors July $25,1963$.

1 This work was supported by National Science Foundation Grant G-18974 while both authors held visiting positions in the University of California, Berkeley. We thank Professor Henry Helson for his kind criticism. 
ties of the inner functions $q$ on $|z|=1$ (i.e., the points at which $q$ is not defined and across which $q$ cannot be continued analytically). This introduces additional complications which are met in $\$ 2$. We indicate at the end possible generalizations of Rudin's theorem.

2. Proof of the theorem. Our proof depends on the structure of invariant subspaces of $H^{\infty}$ determined by us in [6]. We include here a proof of this (in the special case of the circle) for completeness.

Lemma 1. Let $M$ be a weak* closed subspace of $H^{\infty}$ invariant under multiplication by the function $\chi\left(e^{i \theta}\right)=e^{i \theta}$. Then

$$
M=q H^{\infty}
$$

for some inner function $q$.

Proof. Let $M^{\perp}=\left\{f \in L^{1}: \int f g d m=0\right.$ for all $\left.g \in M\right\}$. Then $M^{\perp}$ is a closed subspace of $L^{1}$ with $\chi M^{\perp} \subset M^{\perp}$; the last inclusion is strict, as otherwise we would have $\bar{\chi} M=M$, which is impossible since $M \subset H^{\infty}$. By a known theorem [2], [6], $M^{\perp}$ is of the form $q_{1} H^{1}$, where $q_{1} \in L^{1},\left|q_{1}\right|=1$ a.e. and $H^{1}$ is the $L^{1}$ closure of $A$. Hence $M=q H^{\infty}$, where $q=\chi \bar{q}_{1} . q$ is obviously inner, as $M \subset H^{\infty}$. Thus we proved the lemma.

We need the following for the statement of Lemma 2:

If $f \in H^{\infty}$ and $q \in H^{\infty}$ is an inner function, say that $q$ divides $f$ if $f=q F$ for some $F \in H^{\infty}$. It is known $\left[3\right.$, p. 67] that for every $f \in H^{\infty}$ there exists a unique inner function $q$ such that $q$ divides $f$ and $f / q$ is outer. This $q$ we shall call the inner part of $f$. Given any two inner functions $f, g$ with respectively zeros $\left\{a_{n}\right\},\left\{b_{n}\right\}$ and singular measures $\lambda, \mu$, in their representations (1), any inner function whose zeros are among the common zeros of $f$ and $g$ (with due regard to multiplicity) and whose singular measure $\nu$ is dominated by $\lambda$ and $\mu$ in the sense that $\lambda-\nu \geqq 0$ and $\mu-\nu \geqq 0$, is a common divisor of $f$ and $g$. It is known more generally that

$(\alpha)$ every nonempty set of inner functions has a greatest common divisor [3, p. 85], also [5, Lemma 2].

It is also known that

$(\beta)$ if $f \in A$ and $q$ is an inner function which divides $f$ then $f / q \in A$ $[5$, Lemma 7].

$(\alpha)-(\beta)$ lead to

LEMMA 2. Let $S$ be any nonempty family of functions in $A$, none of which is identically zero and let $K$ be the intersection of the zero sets of these functions. Let $q$ be the g.c.d. of the inner parts of the functions in $S$ whose existence is guaranteed by $(\alpha)$. Then 


$$
S \subset q Z(K) \text {. }
$$

Lemma 3. Let $K$ be as in Lemma 2, and let $[Z(K)]_{*}$ be the weak* closure of $Z(K)$ in $L^{\infty}$. Then $[Z(K)]_{*}=H^{\infty}$.

Proof. Let $q_{1}$ be the g.c.d. of the inner parts of the functions in $Z(K)$. Then by Lemma $2, Z(K) \subset q_{1} Z(K)$. Therefore $q_{1}^{2}$ divides $q_{1}$. Hence $q_{1}=1$. By Lemma $1,[Z(K)]_{*}=q H^{\infty}$ for some inner function $q$. It is easy to see that $q$ divides the inner parts of the functions in $Z(K)$, so $q$ divides $q_{1}$. Hence $q=1$. Q.E.D.

REMARK. It is known from a theorem of Fatou $[3, \mathrm{p} .80]$ that, given any closed set $K$ of Lebesgue measure 0 on the unit circle, there exists a function in $A$ which vanishes precisely on $K$. In view of this, it follows from Lemma 3 that for every closed set $K$ of Lebesgue measure 0 on $|z|=1$, the weak ${ }^{*}$ closure of $Z(K)$ is the whole of $H^{\infty}$. This result is not obvious $a$ priori and seems to be of independent interest.

Proof of the Theorem. Let $q$ and $K$ be as in Lemma 2 with $N$ replacing $S$. Then

$$
N \subset q Z(K) \text {. }
$$

To prove the theorem we have to show that this inclusion is an equality. First we show that.

$$
[N]_{*}=[q Z(K)]_{*}
$$

By Lemma 1,

$$
[N]_{*}=q_{1} H^{\infty}
$$

for some inner $q_{1}$. By Lemma 3, $[q Z(K)]_{*}=q H^{\infty}$. Thus $q_{1} H^{\infty} C q H^{\infty}$, so that $q$ divides $q_{1}$. But $q$ is the g.c.d. of the inner parts of $N$, so $q_{1}$ divides $q$, by (5). Thus (4) is proved.

Suppose now that the inclusion in (3) were strict. Then there exists a regular Borel measure $\mu$ on $X$, orthogonal to $N$, such that

$$
\mu, \operatorname{Lq}_{q} Z(K) \text {. }
$$

Decompose $d \mu$ into its absolutely continuous and singular parts

$$
d \mu=w d m+d \mu_{\text {s. }}
$$

Let $f \in N$. Since $N$ is an ideal and $\mu \perp N$,

$$
\int x^{n} f d \mu=0, \quad n=0,1,2, \cdots
$$

So by the F. and M. Riesz theorem [4], also [3, p. 47], $f d \mu$ is ab- 
solutely continuous, so $f d \mu=f w d m$ and $f d \mu_{s}=0$. Hence $\mu_{s}$ is supported on the null set of $f$. This is true for every $f \in N$. So $\mu_{a}$ is supported on $K$. Therefore

$$
\mu_{\varepsilon} \perp Z(K) \text {. }
$$

In particular, $\mu_{*} \perp N$. Hence $w d m \perp N$, and therefore also $\perp[N]_{*}$ $=[q Z(K)]_{*}$ by (4). In particular, $w d m \perp q Z(K)$. Now trivially by (7) we have $\mu_{s} \perp q Z(K) \subset Z(K)$. Hence $\mu \perp q Z(K)$, contradicting (6). Q.E.D.

The invariant subspace theorem for $L^{\infty}$ proved in Lemma 1 and the proposition $(\alpha)$ are valid for a wide class of algebras $A$ [6], [7], e.g., Dirichlet algebras [3, p. 54]. Beyond this, our proof essentially depends on the F. and M. Riesz theorem in the form in which it is valid for the circle, and the fact $(\beta)$; hence it can be carried over to any Dirichlet algebra in which these are valid. Some algebras for which the F. and M. Riesz theorem in the above form is valid are described in Bishop [1].

\section{REFERENCES}

1. E. Bishop, A general Rudin-Carleson theorem, Proc. Amer. Math. Soc. 13 (1962), 140-143.

2. F. Forelli, Invariant subspaces in $L^{1}$, Proc. Amer. Math. Soc. 14 (1963), 76-79.

3. K. Hoffman, Banach spaces of analytic functions, Prentice-Hall, Englewood Cliffs, N. J., 1962.

4. F. and M. Riesz, Über die Randwerte einer analytischen Funktion, Quatrième Congrés des Math. Scand. (1916), 27-44.

5. W. Rudin, The closed ideals in an algebra of analytic functions, Canad. J. Math. 9 (1957), 426-434.

6. T. P. Srinivasan, Simply invariant subspaces and generalized analytic functions, Proc. Amer. Math. Soc. (to appear).

7. T. P. Srinivasan and Ju-kwei Wang, Weak* Dirichlet algebras, Acta Math. (to appear).

\footnotetext{
University of California, Berkeley, Panjab University, Chandigarh, India and National Taiwan University, Taipei, Formosa
} 